\title{
Part 2: Ultrastructural changes of fibrin networks during three phases of pregnancy: a qualitative investigation
}

\author{
Albe C. Swanepoel ${ }^{1}$, Barend G. Lindeque ${ }^{2}$, Paul J. Swart ${ }^{2}$, Zeelha Abdool ${ }^{2}$, \\ Etheresia Pretorius ${ }^{1}$
}

${ }^{1}$ Department of Physiology, School of Medicine, Faculty of Health Sciences, University of Pretoria, South Africa, ${ }^{2}$ Department of Obstetrics and Gynaecology, Faculty of Health Sciences, School of Medicine, University of Pretoria, South Africa.

Running title: Pregnancy alters the fibrin network morphology.

\section{* Corresponding Author}

Dr A.C. Swanepoel

University of Pretoria

Faculty of Health Sciences

Department of Physiology

Private Bag x323, ARCADIA, 0007, SOUTH AFRICA

Tel: +27 124202535

Fax: +27 124204482

Email: albe.swanepoel@up.ac.za

\section{$\underline{\text { Abstract }}$}

Introduction: Normal pregnancy is characterized by significant alterations in the haemostatic system accompanied by an augmented risk of thrombosis. Materials and methods: The fibrin network ultrastructure of different phases of pregnancy, namely early pregnancy (week $8-14$ ), late pregnancy (week $36-40$ ) as well as post-partum (week 6 - 8 after birth) were compared to non-pregnant fibrin networks as well as each other to establish whether differences in fibrin network morphology exist during pregnancy. Scanning electron microscopy was employed to analyse fibrin network morphology. Results: The fibrin networks from all phases of 
pregnancy appeared similar to each other, exhibiting prominent coagulant formation, an increase in the formation of minor, thin fibers, and the presence of granular globules. Al three phases however differ from the typical fibrin network ultrastructure exhibited by the fibrin networks from non-pregnant individuals. The increase in estrogen associated with pregnancy may cause the increase in coagulation factors and ultimately the pro-thrombotic state characteristic of pregnancy. Conclusions: Since no differences were apparent between the different phases of pregnancy it suggests that activation of the coagulation system commences with pregnancy and this pro-thrombotic state continues till at least 8 weeks after birth. These results may shed light on possible pathological mechanisms employed in the development of abnormal or ailing pregnancy.

Keywords: Pregnancy, Fibrin network, Scanning electron microscopy, Ultrastructure, Thrombosis

\section{Introduction}

A complex chain reaction is initiated when a blood vessel is damaged to curtail the loss of blood and aid the repair of the vessel.(van Geffen and van Heerde, 2012) Primary haemostasis refers to the formation of the primary haemostatic plug comprised of platelets. Secondary haemostasis is the subsequent formation of fibrin strands as a result of complex interactions between plasma coagulation factors. These fibrin fibers strengthen the platelet plug to form the stable, secondary haemostatic plug.(Eyre and Gamlin, 2010)

Normal pregnancy is characterized by significant alterations in the haemostatic system accompanied by an augmented risk of thrombosis. It is specifically during the third trimester that these changes manifest.(Davis, 2000; Hellgren, 2003)

Fibrinogen concentrations may increase up to $200 \%$ above normal levels during pregnancy. This is also true for some coagulation factors. (Almagor and others, 2003; Lockwood, 1999) Thrombin levels are also increased during uncomplicated pregnancy.(de Boer and others, 1989) The concentrations of endogenous anticoagulants however decrease or remain unaffected. These pregnancy-induced 
haemostatic alterations therefore promote the hypercoagulable state associated with pregnancy.(Almagor and others, 2003; Lockwood, 1999)

An elevated coagulability of the blood in conjunction with a decrease in fibrinolytic activity contributes to the prothrombotic state attributed to pregnancy.(Davis, 2000; Hellgren, 2003)

Pregnancy is an independent risk factor for thrombosis. Venous thromboembolism, one of the foremost obstetric causes of maternal death, is estimated to affect almost 1 in 1000 deliveries, with an increased incidence in particularly the third trimester. (Knijff and Goorissen, 2000; Pabinger and Grafenhofer, 2003; Uchikova and Ledjev, 2005)

Thrombotic risk is increased by 4 to 10 fold throughout the gestation period as well as post-partum.(Brenner, 2004) This is attributed to the alterations in haemostatic factors as well as physiological vasodilation associated with pregnancy.(Girling, 2004)

All the changes in the coagulation system during pregnancy form part of an intricate physiological adaptation essential for two related purposes. Firstly the maternal and fetal circulations at the uteroplacental interface can expand during pregnancy without complications. Secondly, during placental separation bleeding from the placental site is sufficiently controlled.(O'Riordan and Higgins, 2003)

The impact of pregnancy on the coagulation system, especially the morphology of the fibrin network, need to be investigated to establish the cause of the increased risk for thrombosis.

\section{Materials and methods}

Participants and sample collection

\section{Pregnancy participants}

Sixty pregnant volunteers were used and two groups were distinguished: the first group included 30 women in the early phase of pregnancy ( $8-14$ weeks) and the second group included 30 women in the late phase of pregnancy (36 - 40 weeks). The second group also participated in the follow-up phase post-partum (6 - 8 weeks 
after birth). Women between the age of 18 and 35 years were employed for the study, and all participant information was handled anonymously. Volunteers for this part of the study were recruited from the Femina Clinic, Pretoria. (Ethical clearance number 185/2011). Informed consent was obtained from each participant.

The participants were non-smokers, did not have a history of thrombotic disease or used any chronic medication known to interfere with coagulation factors and/platelet function. They neither used aspirin or aspirin analogues within 48 hours before sampling.

\section{Control participants}

Thirty control participants were also employed for this study. The control females were also between the age of 18 and 35 years, and all participant information was handled anonymously. Ethical clearance was obtained from the University of Pretoria Ethics Committee (Ethical clearance number 185/2011). Informed consent was obtained from each participant.

The participants were non-smokers, did not have a history of thrombotic disease or used any chronic medication known to interfere with coagulation factors and/platelet function. They neither used aspirin or aspirin analogues within 48 hours before sampling.

\section{Blood collection}

$5 \mathrm{ml}$ of blood were drawn by a qualified nurse from each women participating in the study. Blood was drawn only once from the 30 women in the first pregnancy group. Women forming part of the second group (late pregnancy) had blood drawn on their last visit to the gynaecologist before birth, as well as their first visit again to the gynaecologist post-partum.

$5 \mathrm{ml}$ of blood were drawn by a qualified phlebotomist from each control volunteer. Blood was collected during menses (day $1-5$ of the menstrual cycle), when the levels of all gonadotropic hormones (FSH and $\mathrm{LH}$ ) as well as the ovarian hormones (Estrogen, Progesterone and Inhibin) are at their lowest. [14]

In Part 1 of this article, titled Estrogen causes ultrastructural changes of fibrin networks during the menstrual cycle: a qualitative investigation, pertaining to 
different phases of the menstrual cycle, we established that estrogen influences the fibrin network morphology in a very specific way. We established that the menstrual phase (day $1-5$ of the menstrual cycle) is the best period to draw blood for fibrin network analysis, since this period exhibits typical fibrin network ultrastructure and the effect of elevated estrogen and progesterone that is characteristic of pregnancy can be investigated. (Silverthorn and others, 2009)

Blood was collected in a citrate tube. Whole blood collected in the citrate tube was centrifuged at $1250 \mathrm{rpm}$ for 10 minutes. The plasma supernatant was transferred to an eppindorf tube and centrifuged for a further 4 minutes at $1250 \mathrm{~g}$ to obtain supernatant platelet poor plasma (PPP). The PPP was used to make the fibrin smear for SEM analysis.

\section{Experimental procedures}

\section{Preparation of fibrin clot for Scanning Electron Microscopy analysis}

Fibrin clots were prepared by using human thrombin (provided by The South African National Blood Services). The thrombin is $20 \mathrm{U} / \mathrm{ml}$ and is prepared in biological buffer containing $0.2 \%$ human serum albumin. With the addition of thrombin to the PPP, the conversion of fibrinogen to fibrin is triggered and the liberation of intracellular platelet components including transforming growth factor, platelet derived growth factor and fibroblastic growth factor into the coagulum follows.

A mixture of $10 \mu$ l of PPP (obtained from centrifugation) with $10 \mu$ of human thrombin was made on a glass coverslip to form a fibrin clot (coagulum) on the surface. This glass coverslip containing the coagulum was then placed on filter paper dampened with phosphate buffered solution (PBS). This created a humid environment. The sample was placed at $37^{\circ} \mathrm{C}$ for 5 minutes. Following incubation the samples was placed in PBS and placed on a plate shaker for 20 minutes. This washing process assisted in the removal of any blood proteins possibly ensnared within the fibrin network.

The coverslip with the plasma and thrombin mix was then fixed in a solution of $2.5 \%$ gluteraldehyde for 30 minutes and rinsed three times in $0.075 \mathrm{M}$ sodium potassium phosphate buffer with a $\mathrm{pH}$ of 7.4 for 5 minutes. After rinsing, the sample was left in 
secondary fixative, $1 \%$ osmium tetraoxide $\left(\mathrm{OsO}_{4}\right)$ solution, for 15 minutes. Following fixation, the sample was rinsed again as previously described. The sample was then dehydrated in $30 \%, 50 \%, 70 \%, 90 \%$ and three changes of $100 \%$ ethanol for 5 minutes in each concentration.

The SEM procedures were completed by critical point drying of the material, mounting and coating the sample with carbon and examining the fibrin clot with a Zeiss Ultra plus FEG scanning electron microscope. Photomicrographs were taken at $1 \mathrm{kV}$.

\section{Results}

Figure 1.A-D show micrographs of fibrin networks from non-pregnant participants. These networks are typical of healthy control individuals exhibiting several major, thick fibers and some minor, thin fibers between the thick fibers. The fiber strands are all smooth.
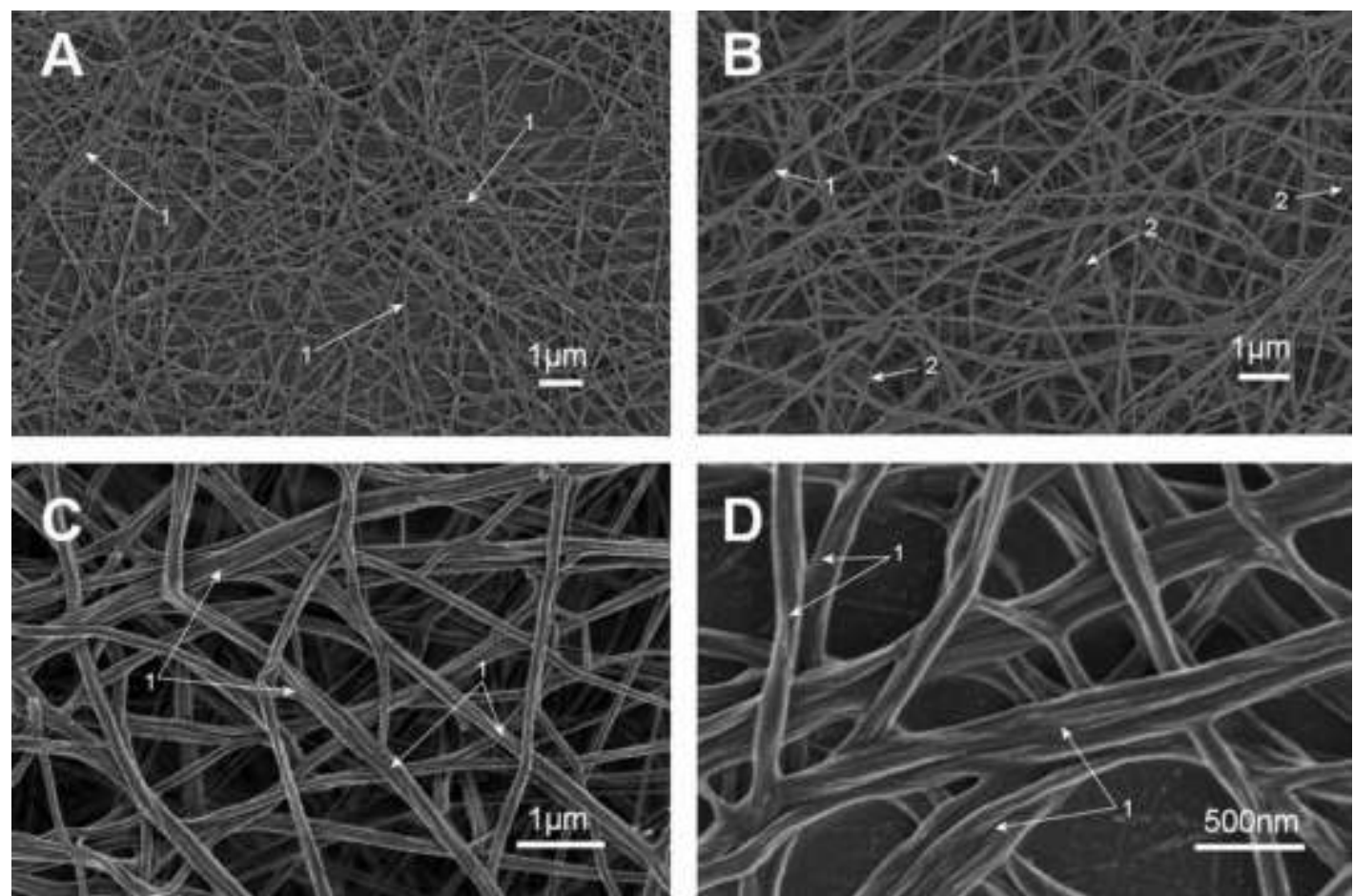

Figure 1. Fibrin networks form control participants.

Machine magnifications: $A=10$ 000x, $B=10$ 000x, C = 40 000x, D = 100 000x.

Major, thick fibers indicated by (1); Minor, thin fibers indicated by (2); Coagulant formation indicated by (3); Protein globular clusters indicated by (4). 
Figure 2.A-D exhibit the fibrin networks from early pregnancy (week 8 - 14) participants. The minor, thin fibers are more prominent and thick, matted layers of coagulant formation can be distinguished. The fibrin strands have a granular morphology and individual granular globules are clearly visible. Plate 1.A-C confirm the altered morphology.
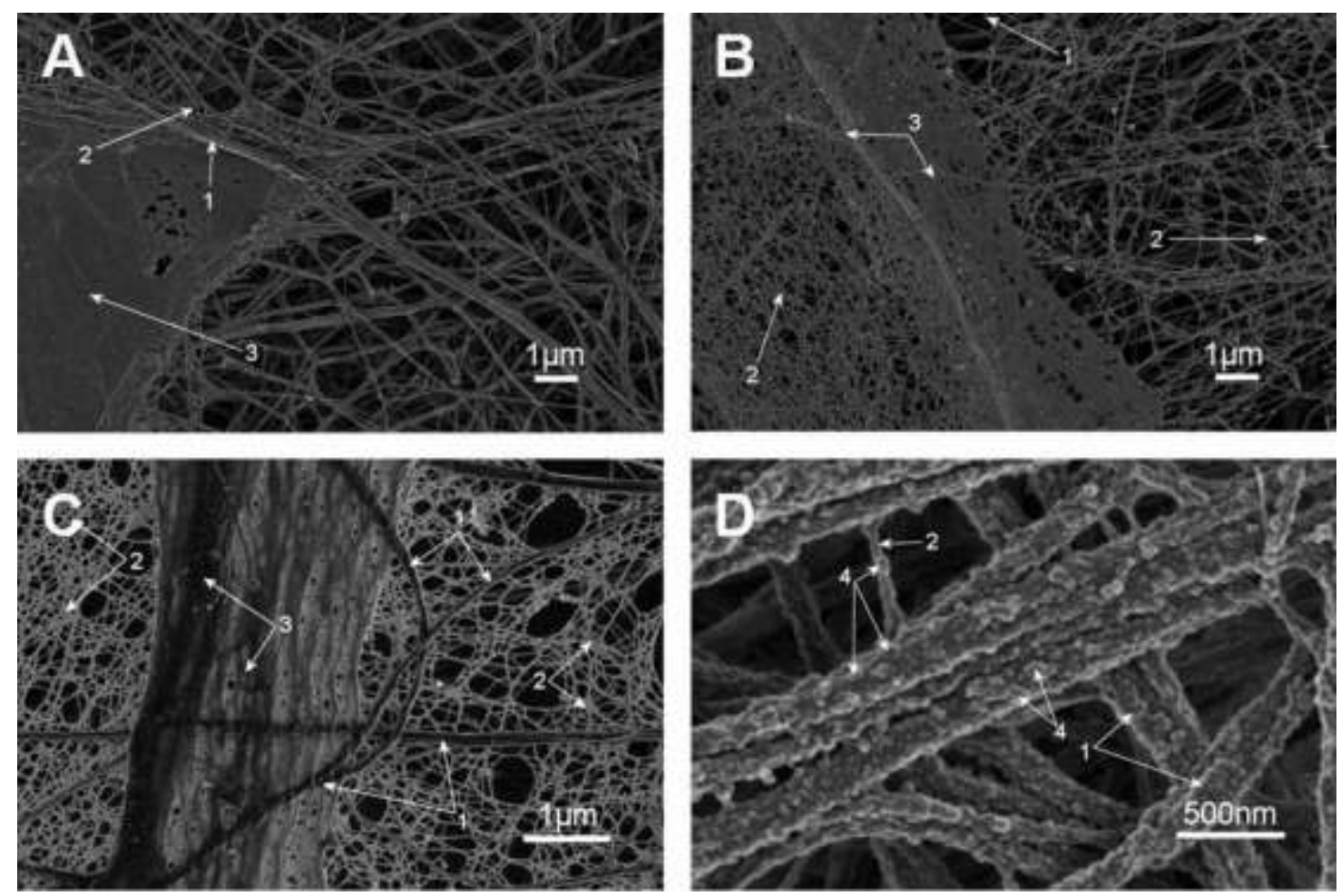

Figure 2. Fibrin network from early pregnancy participants.

Machine magnifications: $A=10000 x, B=10$ 000x, $C=40$ 000x, $D=100$ 000x.

Major, thick fibers indicated by (1); Minor, thin fibers indicated by (2); Coagulant formation indicated by (3); Protein globular clusters indicated by (4).

Figure 3.A-D show the late pregnancy (week $36-40$ ) fibrin networks. Thick, matted coagulant areas are also seen in this group. The minor, thin fibers are also prominent and thickly packed between the major, thick fibers. The same granular globules can be distinguished on the higher magnifications. Plate 2.A-C verify the changes in fibrin network morphology. 

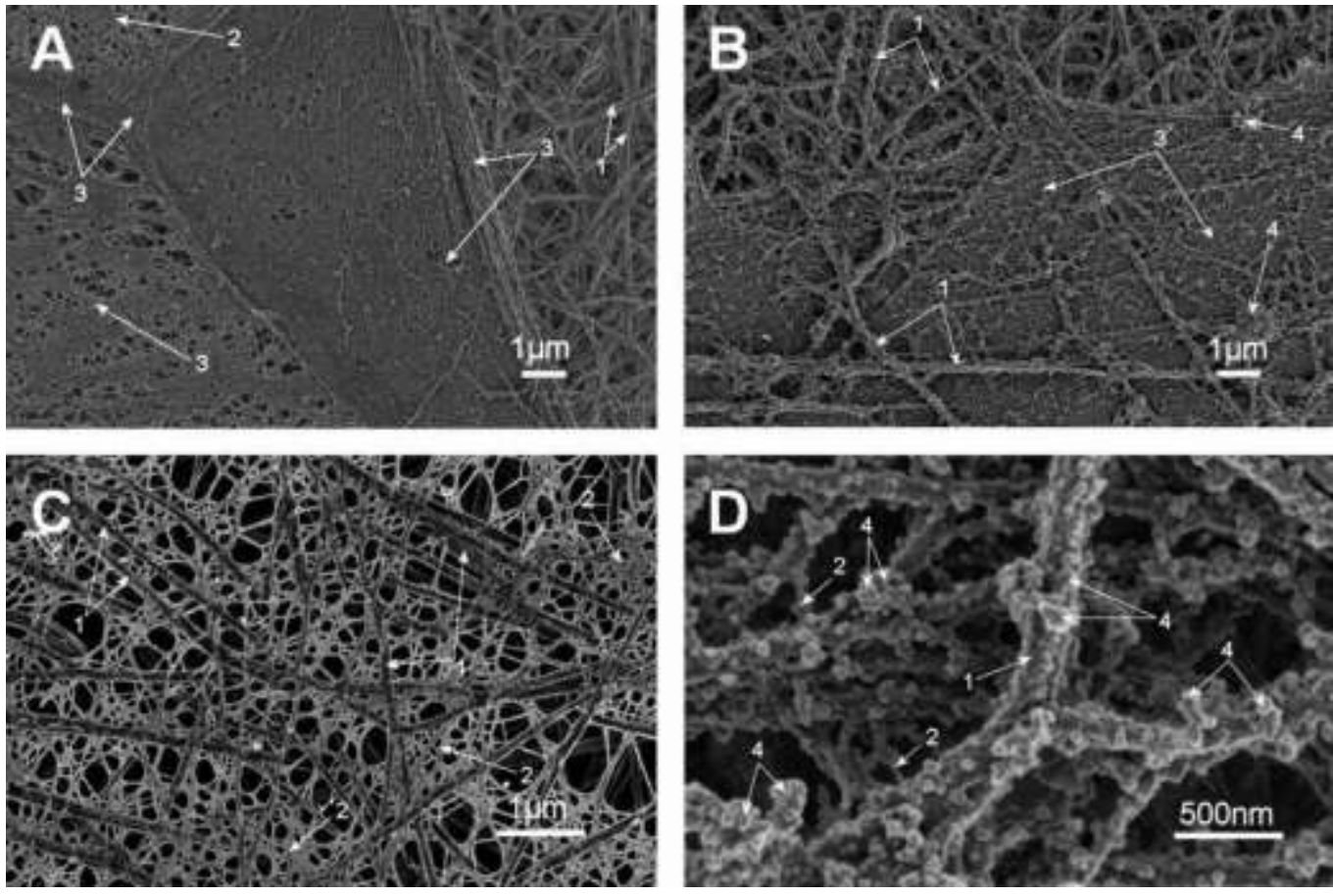

\section{Figure 3. Fibrin network from late pregnancy participants.}

Machine magnifications: $A=10$ 000x, $B=10$ 000x, $C=40$ 000x, D = 100 000x.

Major, thick fibers indicated by (1); Minor, thin fibers indicated by (2); Coagulant formation indicated by (3); Protein globular clusters indicated by (4).
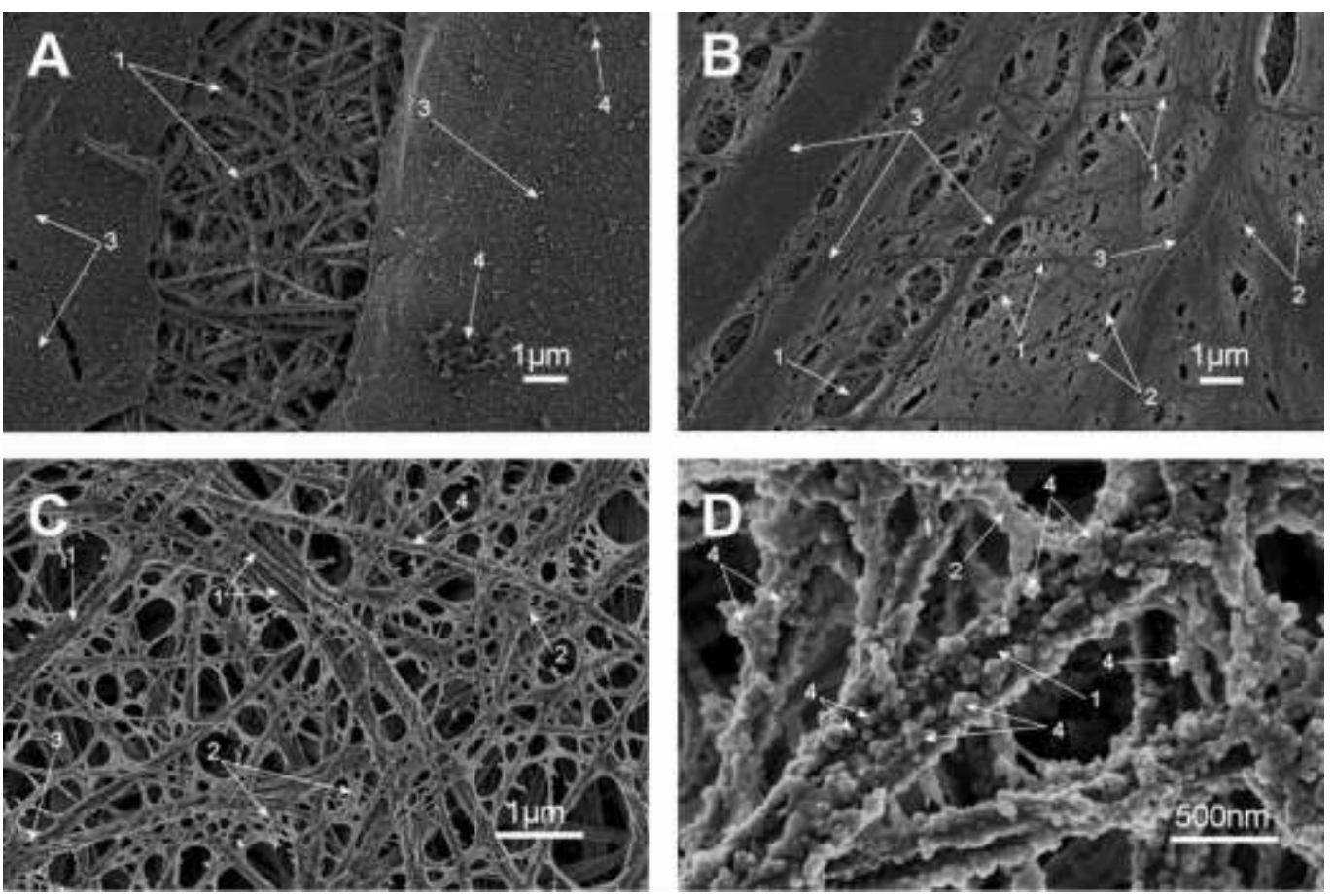

\section{Figure 4. Fibrin network from post-partum participants.}

Machine magnifications: $A=10$ 000x, $B=10$ 000x, $C=40$ 000x, $D=100$ 000x.

Major, thick fibers indicated by (1); Minor, thin fibers indicated by (2); Coagulant formation indicated by (3); Protein globular clusters indicated by (4). 
Figure 4.A-D show fibrin networks from the post-partum group of participants (week 6-8 after birth). Coagulant formation was very prominent. Minor, thin fiber formation between the major, thick fibers is also distinctive. On higher magnification the granular globules seen in the early and late pregnancy groups is once again exhibited. Plate 3.A-C support the variations in ultrastructure.

Overall, the fibrin networks from pregnancy appear similar to each other, exhibiting prominent coagulant formation, an increase in the formation of minor, thin fibers, and the presence of granular globules. Al three phases however differ from the typical fibrin network ultrastructure exhibited by the fibrin networks from non-pregnant individuals.

\section{Discussion}

The fibrin network ultrastructure has been shown to influence the progression of disease like thrombosis and vascular disease.(La Corte and others, 2011) Wolberg emphasised the increasing importance of fibrin network in the investigation of health and disease. (Wolberg, 2012)

Fiber length and diameter is generally directly proportional to each other i.e. increased fiber length is associated with increased fiber diameter. Fiber length and diameter on the other hand is inversely proportional to the degree of branching. Only when branching is minimal will the fibers grow to maximal length and diameter and vice versa.(Weisel, 2007)

The complex relationship between the stiffness of the clot and the fibrin structure is dependent on the length and thickness of the fibers, the density of the network along with the amount of branching and cross-linking between the fibers. It is only when the length, diameter, density and degree of branching of the fibers are in equilibrium that the clot will have maximal rigidity.(Weisel, 2007; Wolberg and Campbell, 2008)

Plasma proteins can directly or indirectly influence fibrin network properties. Alterations in the formation, structure and stability of the fibrin network can occur if the concentration of fibrinogen, fibrin(ogen) binding proteins and thrombin generation is aberrant.(Wolberg, 2010) 
Wolberg recently reviewed fibrin formation, structure and function.(Wolberg, 2012) She found that alterations in normal fibrinogen structure accompanied by increased formation of fibrin strands and greater stability of the network promote thrombosis. When fibrin(ogen) synthesis, formation and function is altered both thrombotic and bleeding disorders can occur.(Wolberg and others, 2012)

Elevated levels of fibrinogen increase the strength and stability of the clot by increasing the density of the fibers as well as the branch points.(Weisel, 2007; Wolberg and others, 2012) Pregnancy is associated with a significant increase in fibrinogen concentration.(Almagor and others, 2003; Lockwood, 1999; Uchikova and Ledjev, 2005) This increased fibrinogen levels during pregnancy may be responsible for the altered fibrin network morphology observed. A greater degree of branching point between the multitudes of minor, thin fibers is evident. The fibrinogen concentration however is not the only possible factor to influence the fibrin network morphology. It most likely the collaboration of various factors that affects the formation of the fibrin network.

Thrombin mediates the conversion of fibrinogen to fibrin to form a haemostatic clot. The fibrin network structure is greatly influenced by the concentration of thrombin available during gelation, since both the thickness of the fiber strands as well as the density of the clot is influenced by thrombin.(Wolberg, 2007; Wolberg and Campbell, 2008)

A very small amount of thrombin in necessary for the cleavage of fibrinopeptides and the subsequent catalyse of fibrin polymerization.(Wolberg, 2007; Wolberg and Campbell, 2008) When thrombin concentrations are low thick, loosely-packed fibrin strands are formed with a high susceptibility to fibrinolysis. High concentrations of thrombin on the other hand will produce thin, tightly-packed fibrin fibers that are not as resistant to fibrinolysis. (Wolberg, 2007; 2010; Wolberg and Campbell, 2008)

Abnormal thrombin generation can lead to variation in fibrin network ultrastructure associated with thrombosis.(Wolberg, 2007)

The quality of the fibrin network determines the binding of thrombin to it. More thrombin is trapped in thin fibrin strands typical of the fibrin network associated with 
the site of vessel injury.(Wolberg, 2007) Thinner fibrin strands additionally trigger increased synthesis of vWF by endothelial cells.(Hamik and others, 2007)

The elevated levels of thrombin associated with pregnancy (de Boer and others, 1989) could therefore be implicated in the ultrastructural changes in the fibrin network seen during pregnancy since a majority of minor, thin fibers are observed. The matted coagulant formation may therefore also be attributed to the increased thrombin concentration. The increased thrombin concentration along with the increase in fibrinogen may play an equal role in the increased formation of the minor, thin fiber network. The thinner fibers, which trap thrombin leading to a greater amount of fibers being formed, also release trigger the release of vWF. The increase in minor, thin fibers may therefore explain the increase in vWF associated with pregnancy.

Lui et al have stated that both coagulation and fibrinolysis are activated during normal pregnancy.(Liu and others, 2009) Fibrinolysis is the breakdown of a fibrin clot. It plays an important role in haemostasis since it prevents occlusion of blood vessels and thus maintaining blood flow. A series of proteins and their specific inhibitors tightly regulate the fibrinolytic system.(La Corte and others, 2011)

Fibrin acts as both cofactor and substrate for plasmin, a fibrinolytic enzyme, during clot dissolution. Therefore the susceptibility of a clot to fibrinolysis is greatly influenced by the fibrin network morphology.(Wolberg, 2007)

Thinner fibrin strands are also more resilient to fibrinolysis than thicker fibrin fibers. Although an individual thick fiber is more slowly lysed than a thin fiber, the clots produced by low thrombin concentrations consist of thick, loosely woven fibrin fibers with fewer individual fibers present for a given amount of fibrinogen. These clots therefore have a greater susceptibility to lysis than tightly-packed thin fibers, with subsequently more individual fibers per fibrinogen, produced by higher thrombin concentrations. While plasmin generation occurs on individual fibers, the rate of plasmin activity is limited by the plasmin movement through the three-dimensional fibrin network.(Blombäck and others, 1994; Collet and others, 2000; Wolberg, 2007; 2010) 
The configuration and especially the number of fibers per volume in a fibrin clot therefore have a greater influence on the rate of fibrinolysis than the fibers as such. It is particularly a fibrin network that is composed of a large amount of thin fibers that readily binds rtPA. The bound rtPA then diffuses into the clot at a slower rate compared to a network consisting of a smaller amount of thicker fibrin strands. (Collet and others, 2003)

The increased incidence of minor, thin fibers associated with pregnancy therefore influences fibrinolytic activity. The rate of fibrinolysis is decreased since the network is closely packed and plasmin movement through the three-dimensional network is limited.

The increased fibrinogen and thrombin concentrations result in the formation of a matted, tightly-packed network of mainly minor, thin fibers which are more resilient to fibrinolysis. These changes in fibrin network ultrastructure therefore increase the risk of thrombosis, since fibrinolysis is not able control the coagulant formation.

Venous thrombosis or thromboembolism is characteristic of plasma hypercoagulability. Triggered expression of procoagulant activity on intact endothelium is the proposed mechanism. This can be caused by inflammation or reduced blood flow after prolonged immobility.(Wolberg and others, 2012)

Venous and/or arterial coagulopathies have been associated to various haematological factors including irregular pro- and anticoagulant protein levels, thrombin concentration, the activity of clotting factors or the resistance of these clotting factors to activation and fibrinolysis inhibitors. (Wolberg and others, 2012)

Increased levels of fibrinogen are an invariable risk factor for venous and arterial thrombosis.(Wolberg and others, 2012) Increased levels of prothrombin also increase the risk of venous thrombosis.(Poort and others, 1996)

In pregnancy the risk for venous thrombosis augmented. A six-fold increase in incidence of venous thromboembolism is associated with pregnancy.(Holmes and Wallace, 2005)

The variation in fibrin ultrastructure observed in pregnancy may explain how thrombin and fibrinogen increase the risk of venous thromboembolism. The network 
of minor, thin fibers trap additional thrombin, laying down more layers of fibrin originating from the increased amount of fibrinogen and may ultimately occlude a vessel. The proposed mechanism of venous thrombosis where procoagulant activity is trigger on the intact endothelial wall of a blood vessel could be ascribed to the prothrombotic condition of the fibrin network observed in this study.

In 2009 Pretorius et al compared the fibrin networks from a healthy pregnant individual and a pregnant individual with dysfibrinogenaemia using scanning electron microscopy. Samples were taken at 30 weeks as well as 3 months post-partum. The fibrin networks of both individuals displayed a fine, dense net of minor, thin fibers distributed evenly over the major, thick fibers. And since this net was not present in healthy non-pregnant individuals or non-pregnant individuals with dysfibrinogenaemia, these changes were specific to pregnancy. Three months postpartum the morphology of the fibrin network returned to normal. Pretorius and her colleagues suggested that this altered morphology could contribute to increased risk of thrombosis since the denser network of minor, thin fibers may influence the rate fibrinolysis.(Pretorius and others, 2009)

It is evident from the SEM micrographs that apparent morphological changes can be seen in early pregnancy, late pregnancy and are still evident 6 - 8 weeks postpartum. This indicates that the coagulation system is activated from the commencement of pregnancy up until at least 8 weeks after birth.

Hormonal changes, especially an increase in estrogen, increase the activity of certain coagulation factors such as XII, VIII, X and IX. [28] The increase in coagulation factors brought on by the increase in estrogen during pregnancy could be responsible for the formation of granular globules on the fibrin strands.

When thrombin converts fibrinogen to fibrin it cleaves fibrinopeptides from the central domain. This exposes knobs that can then interact with holes that are always exposed at the ends of the molecule.(Weisel, 2005) It may be that the exposed knobs themselves extent or aggregate to give rise to the granular globules or the altered fibrin structure could yield areas to which clusters of coagulation proteins more readily attach to the fibrin strand. 
The same granular globules were seen during different phases of the menstrual cycle. It was concluded that estrogen causes the alterations in the internal and external structure of the fibrin strands since it coincide with estrogen peaks in the menstrual cycle.

In conclusion, the coagulation as well as fibrinolytic system is activated during pregnancy. Several procoagulant factors, including fibrinogen, are increased along with elevated concentrations of thrombin and VWF and a decrease in fibrinolytic activity. The increase in estrogen associated with pregnancy may cause the increase in coagulation factors and ultimately the pro-thrombotic state characteristic of pregnancy.

The fibrin networks from pregnancy exhibit a noticeable increase in the formation of minor, thin fibers and prominent coagulant formation. Large quantities of granular globules are also present. Al three phases differ from the typical fibrin network ultrastructure consisting of mainly major, thick fibers and a few minor, thin fibers. There are no apparent differences between early phase, late phase or post-partum fibrin networks. This suggests that activation of the coagulation system commences with pregnancy and this pro-thrombotic state continues till at least 8 weeks after birth.

This is the first morphological investigation of fibrin networks during normal pregnancy. These physiological changes observed during normal pregnancy can be used as a standard of comparison to other diseased states found during pregnancy. These results may shed light on possible pathological mechanisms employed in the development of abnormal or ailing pregnancy.

\section{Ethical considerations}

Ethical clearance was obtained from the Human Ethics Committee from the University of Pretoria. All individuals completed informed consent forms.

\section{$\underline{\text { Acknowledgements }}$}

The authors acknowledge Ampath (Drs Du Buisson, Kramer, Swart, Bouwer Inc.) for their assistance with blood collection. 


\section{Conflict of interest}

The authors have no conflict of interest to declare.

\section{References}

Almagor M, Gorten D, Yaffe H. 2003. Decreased levels of fibrinogen in very high parity women. European Journal of Obstetrics \& Gynecology and Reproductive Biology 106(2): 148-150.

Blombäck B, Carlsson K, Fatah K, Hessel B, Procyk R. 1994. Fibrin in human plasma: gel architectures governed by rate and nature of fibrinogen activation. Thrombosis Research 75(5): 521-538.

Brenner B. 2004. Haemostatic changes in pregnancy. Thrombosis Research 114(5): 409-414.

Collet J-P, Lesty C, Montalescot G, Weisel JW. 2003. Dynamic changes of fibrin architecture during fibrin formation and intrinsic fibrinolysis of fibrin-rich clots. Journal of Biological Chemistry 278(24): 21331-21335.

Collet J, Park D, Lesty C, Soria J, Soria C, Montalescot G, Weisel J. 2000. Influence of Fibrin Network Conformation and Fibrin Fiber Diameter on Fibrinolysis Speed Dynamic and Structural Approaches by Confocal Microscopy. Arteriosclerosis, thrombosis, and vascular biology 20(5): 1354-1361.

Davis GL. 2000. Hemostatic changes associated with normal and abnormal pregnancies. Clinical laboratory science : journal of the American Society for Medical Technology 13(4): 223-228.

de Boer K, ten Cate JW, Sturk A, Borm JJ, Treffers PE. 1989. Enhanced thrombin generation in normal and hypertensive pregnancy. American journal of obstetrics and gynecology 160(1): 95-100.

Eyre L, Gamlin F. 2010. Haemostasis, blood platelets and coagulation. Anaesthesia \& Intensive Care Medicine 11(6): 244-246.

Girling JC. 2004. Physiology of pregnancy. Anaesthesia \& Intensive Care Medicine 5(7): 215-218.

Hamik A, Lin Z, Kumar A, Balcells M, Sinha S, Katz J, Feinberg MW, Gerszten RE, Edelman ER, Jain MK. 2007. Kruppel-like factor 4 regulates endothelial inflammation. Journal of Biological Chemistry 282(18): 13769-13779. 
Hellgren M. Hemostasis during normal pregnancy and puerperium2003. New York: Stratton Intercontinental Medical Book Corporation, c1974-. p 125-130.

Holmes V, Wallace J. 2005. Haemostasis in normal pregnancy: a balancing act? Biochemical Society Transactions 33(2): 428.

Knijff S, Goorissen E. 2000. Summary of contraindications to oral contraceptives: Parthenon Publishing Group.

La Corte AC, Philippou H, Ariëns R. 2011. Role of fibrin structure in thrombosis and vascular disease. Adv Protein Chem Struct Biol 83: 75-127.

Liu X-h, Jiang Y-m, Shi H, Yue X-a, Wang Y-f, Yang H. 2009. Prospective, sequential, longitudinal study of coagulation changes during pregnancy in Chinese women. International Journal of Gynecology \& Obstetrics 105(3): 240-243.

Lockwood CJ. 1999. Heritable coagulopathies in pregnancy. Obstetrical \& gynecological survey 54(12): 754.

O'Riordan MN, Higgins JR. 2003. Haemostasis in normal and abnormal pregnancy. Best Practice \& Research Clinical Obstetrics \& Gynaecology 17(3): 385-396.

Pabinger I, Grafenhofer H. 2003. Pregnancy-associated thrombosis. Wiener Klinische Wochenschrift 115(13-14): 482-484.

Poort SR, Rosendaal FR, Reitsma PH, Bertina RM. 1996. A common genetic variation in the $3^{\prime}$-untranslated region of the prothrombin gene is associated with elevated plasma prothrombin levels and an increase in venous thrombosis. Blood 88(10): 3698-3703.

Pretorius E, Bronkhorst P, Briedenhann S, Smit E, Franz RC. 2009. Comparisons of the fibrin networks during pregnancy, nonpregnancy and pregnancy during dysfibrinogenaemia using the scanning electron microscope. Blood Coagulation \& Fibrinolysis 20(1): 12-16.

Silverthorn DU, Ober WC, Garrison CW, Silverthorn AC, Johnson BR. 2009. Human physiology: an integrated approach: Pearson/Benjamin Cummings.

Uchikova EH, Ledjev II. 2005. Changes in haemostasis during normal pregnancy. European Journal of Obstetrics \& Gynecology and Reproductive Biology 119(2): 185-188.

van Geffen M, van Heerde WL. 2012. Global haemostasis assays, from bench to bedside. Thrombosis Research 129(6): 681-687. 
Weisel JW. 2005. Fibrinogen and Fibrin. In: Advances in Protein Chemistry. David ADP, John MS, editors: Academic Press. pp 247-299.

Weisel JW. 2007. Structure of fibrin: Impact on clot stability. Journal of Thrombosis and Haemostasis 5(SUPPL. 1): 116-124.

Wolberg AS. 2007. Thrombin generation and fibrin clot structure. Blood Reviews 21(3): 131-142.

Wolberg AS. 2010. Plasma and cellular contributions to fibrin network formation, structure and stability. Haemophilia 16(s3): 7-12.

Wolberg AS. 2012. Determinants of fibrin formation, structure, and function. Current opinion in hematology 19(5): 349-356.

Wolberg AS, Aleman MM, Leiderman K, Machlus KR. 2012. Procoagulant activity in hemostasis and thrombosis: Virchow's triad revisited. Anesthesia and analgesia 114(2): 275.

Wolberg AS, Campbell RA. 2008. Thrombin generation, fibrin clot formation and hemostasis. Transfusion and Apheresis Science 38(1): 15-23. 